\title{
Role of Critical Care Units in the management of obstetric patients (Review)
}

\author{
KONSTANTINOS KOUKOUBANIS ${ }^{1}$, ANASTASIA PRODROMIDOU ${ }^{1}$, EMMANOUIL STAMATAKIS ${ }^{2}$, \\ DIMITRIOS VALSAMIDIS ${ }^{2}$ and NIKOLAOS THOMAKOS ${ }^{1}$ \\ ${ }^{1}$ First Department of Obstetrics and Gynecology, Medical School, National and Kapodistrian University of Athens, \\ 'Alexandra' Hospital; ${ }^{2}$ Department of Anesthesiology and Pain Management, \\ 'Alexandra' General Hospital of Athens, 11528 Athens, Greece
}

Received December 16, 2020; Accepted April 19, 2021

DOI: $10.3892 /$ br.2021.1434

\begin{abstract}
Pregnancy is considered a natural process for the majority of women. However, a limited proportion of pregnancies and deliveries can present with a broad variety of complications that may require admission to a Critical Care Unit (CCU). In the present review, the indications of admission of obstetrical and postpartum patients to CCUs were critically evaluated with a particular focus on the management of their complications. The management of critically ill obstetric patients remains challenging due to the physiological changes that occur during pregnancy, pregnancy-related diseases and the need to carefully consider the well-being of the fetus before any intervention can be recommended/performed. Indications for admission to CCUs include both obstetric and non-obstetric conditions that may require continuous monitoring and further interventions. Hypertensive disorders of pregnancy and mass hemorrhage are amongst the most common causes of admission to CCUs in pregnant and postpartum women. The establishment of a diagnostic and care algorithm based on the contribution of a multidisciplinary team is of critical importance to aid in the determination of which patients will require intensive care, and to assist in deciding what type of critical care each critically ill patients receives.
\end{abstract}

\section{Contents}

1. Introduction

2. Materials and methods

3. Epidemiology

4. Levels of maternal care

5. Anatomical and physiological changes in pregnancy

Correspondence to: Dr Anastasia Prodromidou, First Department of Obstetrics and Gynecology, Medical School, National and Kapodistrian University of Athens, 'Alexandra' Hospital, Lourou 2, 11528 Athens, Greece

E-mail: a.prodromidou@hotmail.com

Key words: critical care, intensive care unit, critically ill, obstetric complications
6. Indications of admission

7. Post-treatment course - scoring systems

8. Conclusions

\section{Introduction}

The vast majority of pregnancies are completed without any notable complications. Advances in medical care across the world, and the broader access to healthcare systems has lead to a significant decrease in maternal mortality globally (1). However, a limited proportion of pregnancies and deliveries present with a broad variety of complications that may require admission to a Critical Care Unit (CCU), including Intensive Care Units (ICUs) and High Dependency Units (HDUs). The altered physiology that occurs during pregnancy, potential hazardous effects of certain drugs along with the limitations in performing some interventions due to the need to consider the effects on the fetus, can hinder the management of critically ill pregnant patients (2). Hemorrhagic conditions, hypertensive diseases and sepsis are considered the predominant complications that can lead to death (1). Additionally, another factor that should be addressed, for patients that are finally admitted to a CCU, is the relative lack of experience in the management of these unique conditions, from both the staff and anesthesiologists. Therefore, it has been suggested that specialized obstetrical CCUs should be established and a multidisciplinary team should be aware of the management needs of critically ill pregnant and postpartum patients with complications (3). Critical care during the pregnancy and postpartum period is based primarily on the recommendations derived from non-pregnant critical care patients, due to the limited data available on the obstetric critically ill patients (4).

The aim of the present review is to critically review the indications of admission of obstetrical and postpartum patients to CCUs, and to highlight the unique aspects of management of the patients with obstetrical complications that are admitted to CCUs.

\section{Materials and methods}

A thorough search of the currently available literature using three electronic databases (PubMed, Google Scholar and Scopus) was performed. Articles published up to November 2020 that 
presented information with regard to pregnant and postpartum patients in need of intensive care were evaluated along with the references of the eligible articles, and all the relevant articles were retrieved as the full text. The following keywords were utilized: 'Intensive Care Unit', 'Critical Care Unit', 'obstetric complication', 'postpartum', 'indications' and 'maternal care'. Prospective and retrospective original articles (comparative and non-comparative), case reports and reviews that were written in English language were assessed and critically appraised.

\section{Epidemiology}

The maternal mortality ratio (MMR) is defined as the number of mothers that die per every 100,000 births (5). It serves as a significant indicator of the quality of care provided by health services to the mother and child (6). The annual MMR in the United Sates during 2013 was 28 maternal deaths per 100,000 births, which has doubled from the 12 per 100,000 that was recorded in 1990 (7). This can be potentially attributed to the significant increase in the rates of cesarean sections and the increase in age at conception, which in combination with the broader applications of assisted reproduction technologies, result in higher-risk pregnancies in patients of advanced reproductive age who are more likely to present with comorbidities (8). In 2008, the MMR in developing countries was $15 x$ higher compared with the developed world (9). This discrepancy can be attributed to the insufficient follow up, the lack of appropriate health services and the considerably poorer living conditions in those countries (9).

In 2009 the World Health Organization (WHO) introduced the terms 'near misses', 'severe maternal morbidity' (SMM) and 'severe associated maternal morbidity' (SAMM) to indicate the advanced level of health care that would be required for critically ill patients (10). The prevalence of such cases was estimated at 12.9 cases per 1,000 deliveries and 2.9 per 1,000 cases of postpartum hospitalizations (11). The number of CCU admissions has been proposed as an indicator of the quality of maternal health (8). The respective admissions have been reported to range from 0.5-4.2 per 1,000 deliveries in developed countries (8).

For patients admitted to CCUs, $63-92 \%$ were in the postpartum period, whereas the median length of CCU stay for women admitted antepartum and postpartum was 2 and 1.1 days, respectively (4). Furthermore, the proportion of pregnant patients admitted to CCUs ranged from 3.3-14\%, with the lowest rates observed in developed countries with well-organized health care systems (12). Interestingly, it has been shown that $\sim 50 \%$ of maternal deaths can be prevented through early recognition and intervention in critically ill patients who require admission to a $\mathrm{CCU}$ (13).

\section{Levels of maternal care}

In an attempt to reduce maternal morbidity and mortality, the levels of maternal care are constantly improving, to ensure the necessary care is provided to women in the peri-partum period. According to levels of maternal care proposed by a UK Department of Health, the care that a patient receives in the delivery suite is stratified into four levels based on the support and interventions required as follows; Level 0 , care that is provided to low risk women in the general ward; level 1, care which requires non-invasive patient observation and monitoring; level 2, organ support for a single organ is required, including support of the respiratory, cardiovascular, neurological or hepatic systems; and level 3 refers to women in need of mechanical ventilation for respiratory support or support of two or more organs (14). The aforementioned levels can be used to decide which ward a woman with complications is admitted to as follows: Level 0 , patients are hospitalized in the general ward; levels 1 and 2 patients are admitted to the HDU; and level 3 care should be provided in the ICU (14). Excluding the obstetricians/gynecologists; specialists of other fields of medicine may not be as familiar with the physiological changes that occur during pregnancy, and thus the degree of involvement of any underlying conditions these patients may have may be underestimated. Therefore, it is critical that the HDU should be staffed by obstetricians, anesthesiologists, fetal maternal medicine specialists, neonatologists, midwives and physiotherapists. With regards to the ICU, a multidisciplinary team is also of paramount importance for an integrated and accurate approach (15). In particular, the care of the obstetrical patient necessitates knowledge on the physiology of two patients (the mother and the fetus) and for critically ill patients the co-operation of different specialists, including intensivists, anesthesiologists, obstetricians/gynecologists, Fetal Maternal Medicine (FMM) specialists and neonatologists is required (8).

\section{Anatomical and physiological changes in pregnancy}

Pregnancy as a condition is characterized by a variety of anatomical and physiological changes, which complicate the assessment and management of pregnant women. To that end, knowledge of the anatomical and physiological alterations that occur during pregnancy is appropriate so as to enable the optimal management of critically ill pregnant patients in need of admittance to a CCU. First, with regard to the changes in the cardiovascular system, an increase in cardiac output by $30-50 \%$ has been observed, and is attributed to the increase in both the stroke volume and the heart rate, which can further worsen pre-existing cardiac comorbidities (16). In addition to this, the elevation in progesterone and estrogen levels results in a drop in the peripheral vascular resistance, which in combination with the enlargement of the uterus, can compress the aorta (16). Consequently, the increased cardiac afterload decreases the cardiac return, and this can result in hypotension, which can present with the known vena cava syndrome (17). Moreover, the increase in the volume of plasma results in a $30-50 \%$ elevation in total blood volume, whereas the respective increase in the quantity of red blood cells is less significant. Those two factors primarily account the development of an anemic state, termed 'anemia of pregnancy' (2). Concerning the coagulation, an increase in coagulative factors, such as thrombin and fibrinogen, with a simultaneous decrease in anticoagulation factors, such as anti-thrombin and protein S, predispose a pregnant woman to a hypercoagulative environment (18).

The enlargement of the uterus and the subsequent elevation of the diaphragm represent the primary causes of a decrease in functional residual capacity (19). This decrease, combined with the increased oxygen consumption can lead to hypoxia (20). Due to the increase in progesterone levels, the elevation in tidal volume and ventilation minute volume leads to a decrease in the $\mathrm{PaCO}_{2}$ and subsequent respiratory alkalosis (21). These changes can differentiate the arterial blood gas values with an 
Table I. Primary indications for admission to an intensive care unit in obstetric patients.

Non-obstetric indications

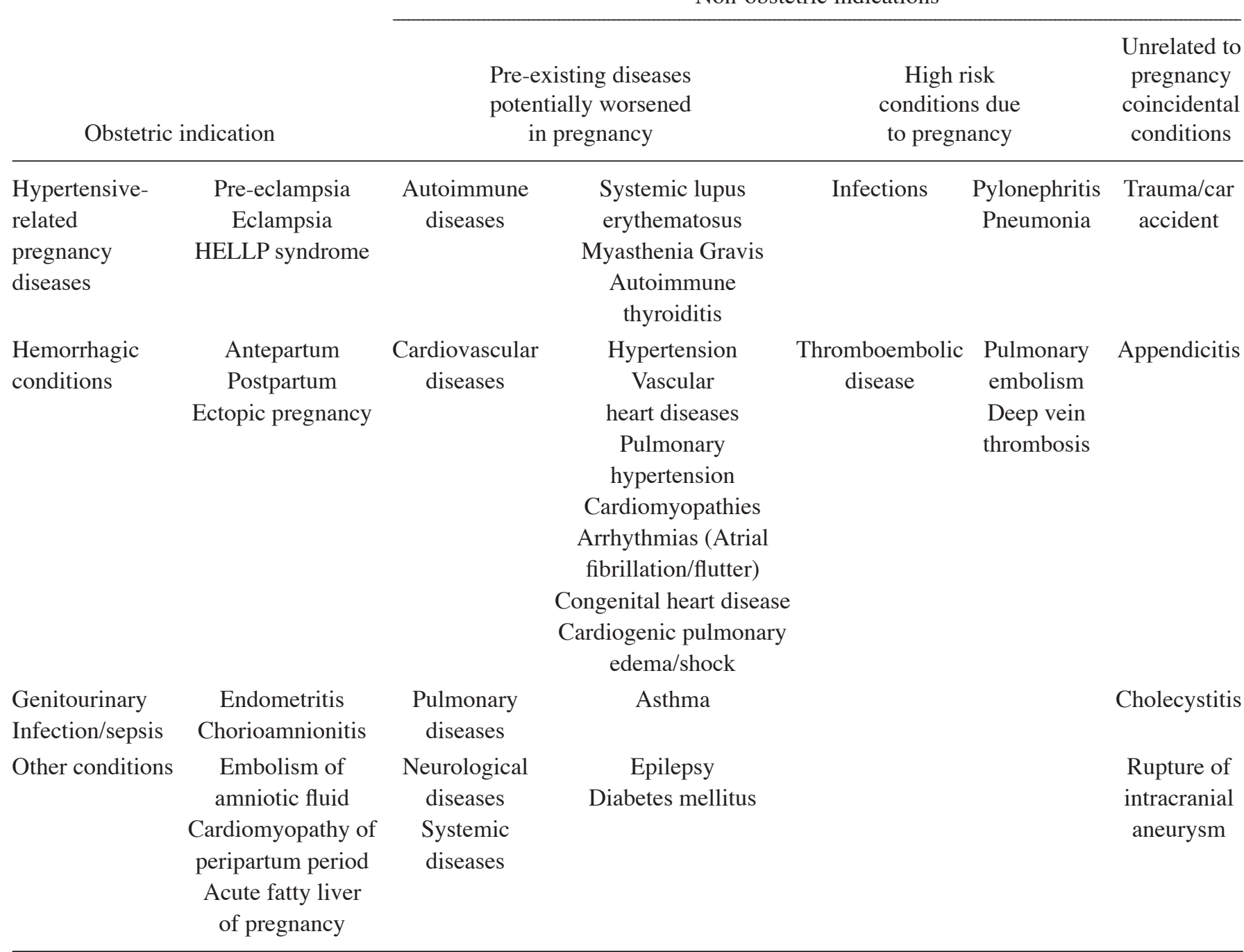

HELLP, hemolysis, elevated liver enzymes, and low platelet.

alkalotic PH (20). Laryngeal edema and nasal congestion are frequently observed, and in combination with the weight gain and breast enlargement, can make tracheal intubation more challenging compared with non-pregnant patients (22).

Renal function is also modified during pregnancy, to accommodate for the gestational requirements and maintain a physiological electrolyte composition; the alterations in the tubal function result in restoration of increased amounts of nutrients and electrolytes, including glucose, proteins and amino acids, so as to be available to be passed on to the fetus (23). The creatinine clearance is increased during pregnancy to levels of $120-160 \mathrm{ml} / \mathrm{min}$, whereas the levels of serum creatinine are decreased to $0.4-0.7 \mathrm{mg} / \mathrm{dl}$, which approximately translates to $77-84 \%$ of the normal values in non-pregnant women, and may be primarily attributed to gestational hyperfiltration $(20,24)$.

Finally, the delayed gastric emptying and the relaxation of the esophageal sphincter are responsible for the feeling of heartburn that is a common complaint of pregnant women (2). Additionally, it has been stated that due to prolonged gastric emptying and delayed motility, there is a possibility of alterations to the pharmacokinetics of orally administered medicines (2). The aforementioned changes can be attributed to the significant increase in estrogen and progesterone levels (21). The progesterone-induced gastric changes increase the risk of aspiration during endotracheal intubation (21). Thus, epidural anesthesia is preferable to general anesthesia, when possible.

\section{Indications of admission}

Patients who are admitted to CCUs may suffer from both obstetric and non-obstetric conditions that may require continuous monitoring (invasive and non-invasive) and further interventions (summarize in Table I).

The non-obstetric conditions that may complicate and may be further worsened during pregnancy include immune diseases (Systemic lupus erythematous, myasthenia Gravis and autoimmune thyroiditis), cardiovascular diseases (hypertension, valvular heart diseases, pulmonary arterial hypertension, cardiomyopathies, arrhythmias and congenital heart disease), respiratory and neurological conditions (such as asthma and 
epilepsy), and systemic diseases (such as diabetes mellitus. Conversely, the non-obstetric conditions that are associated with pregnancy can be attributed to the physiological and anatomical changes that come with pregnancy, infectious diseases (pyelonephritis and pneumonia) thromboembolic diseases (deep vein thrombosis and pulmonary embolism) and pulmonary edema, all of which have been addressed above. Finally, some additional diseases that can arise in pregnancy, but are coincidental causes of CCU admission include trauma, appendicitis, cholecystitis and viral infections, such as H1N1 infection (25).

With regards to the obstetric indications for admission to CCUs they are defined as follows: Hypertensive disorders of pregnancy, including preeclampsia and hemolysis, elevated liver enzymes and low platelet count (HELLP) syndrome, and these are considered the most prevalent causes of CCU admission in pregnant women (26). They represent both severe conditions that can have detrimental effects on the health of both mother and the fetus, as well as being associated with significantly high morbidity and mortality rates. HELLP syndrome may imitate a plethora of non-obstetric disorders (27). Diagnosis is based on the clinical symptoms including hypertension $(15 \%$ of patients with HELLP syndrome exhibit blood pressure within normal ranges), malaise, nausea and epigastric pain (28), along with the laboratory findings of microangiopathic hemolysis (schistocytes, peripheral swear, low serum hemoglobin and elevated lactate dehydrogenase), elevated levels of serum glutamic-oxalacetic transaminase/serum glutamic-pyruvic transaminase (up to $70 \mathrm{IU} / \mathrm{l})$ and low platelets $(<100,000 / \mu \mathrm{l})$. Additionally, eclampsia is the most severe manifestation of hypertensive disorders in pregnancy, and is defined as the onset of tonic-clonic, focal or multifocal seizures in the absence of other causative conditions, such as epilepsy, cerebral arterial ischemia and infarction, intracranial hemorrhage or drug use (29). Injection of magnesium sulfate is used to prevent eclamptic seizures by neuromuscular blockade or central action. The use of magnesium requires monitoring for potential toxicity, which includes observation of urine output, vital signs and respiratory rate, as well as response of the patellar reflex and surveillance of the serum magnesium levels, which physiologically range from 4-7 mEq/l (30). Magnesium toxicity, which can occur in cases of elevated magnesium levels is another indication of admission to CCUs, and dialysis to clear magnesium may be required (31). For critically ill patients with preeclampsia conditions, fluid management serves a key role. A strict fluid balance should be maintained, aiming to simultaneously limit the risk of both pulmonary edema potentially caused by fluid overload and renal failure, which can be caused by volume restriction (32). A rate of $80 \mathrm{ml} / \mathrm{h}$ intravenous fluid administration has been proposed by certain studies, although there is insufficient evidence in this field (32). The final treatment for the aforementioned conditions is to proceed with the delivery as soon as possible, whilst simultaneously taking into account the viability of the fetus and the ability to administer corticosteroids to ensure lung maturation (27).

Massive hemorrhaging and the subsequent hypovolemic shock are also considered amongst the leading causes for admission to CCUs, amongst the obstetric population. Massive hemorrhaging can occur either during the antepartum period primarily due to a ruptured ectopic pregnancy, placental abruption or pathological invasion of the placenta (placenta accreta), or during the postpartum course (27). Postpartum hemorrhage (PPH) is a primary cause of maternal mortality (30). Major obstetric hemorrhage is defined as blood loss of $>1,500 \mathrm{ml}$ of blood, or blood loss with signs of shock (33). Atonic uterus is the primary cause of PPH and is attributed to the 4Ts rule: Tone, Trauma, Tissue-placenta remnants and Thrombin (34). Due to the severity of the underlying condition of the patient, continuous monitoring of the vital signs along with concomitant transfusion of blood products is highly recommended in cases where the hemoglobin levels are $<8 \mathrm{~g} / \mathrm{dl}$ or where the patients exhibits impaired coagulation, so as to ensure hemodynamic stability. Additional interventions to manage the cause of the hemorrhage should be performed, and include administration of oxytocin $10 \mathrm{U} / \mathrm{h}$, ergometrine $0.5 \mathrm{mg}$ through intravenous or intramuscular injections and misoprostol per rectum (maximum dose $800 \mathrm{mg}$ ) combined with uterine massaging. The aforementioned treatments are considered the optimal modalities to manage a patient with an atonic uterus and hemorrhage, who is at risk of hypovolemic shock and collapse (35). In cases of failure of these approaches, uterine artery ablation and caesarean hysterectomy are the final resorts used to prevent maternal death as a result of the severe hemorrhage (36).

Amniotic fluid embolism (AFE) or anaphylactic syndrome of pregnancy is a rare but severe condition that is associated with significantly elevated mortality rates of $\sim 80 \%$. The pathogenetic pathway involves an inflammatory anaphylactoid response to fetal antigens, which occurs due to the disruption in the maternal-fetal barrier at delivery and leads to increased pulmonary and/or systemic vascular resistance, decreased left ventricular function and coagulopathy. The patient presents clinical signs of respiratory failure and cardiogenic shock with severe hypoxia, hemodynamic collapse and coagulopathy $(37,38)$. There is no gold-standard approach for the diagnosis of the disease, and in the majority of cases, the diagnosis is based on clinical signs and symptoms (39). The management of AFE is primarily supportive, and includes cardiopulmonary resuscitation, administration of fluids and blood products for the correction of coagulopathy intravenously, administration of anticoagulant agents, and oxygen support or mechanical ventilation in cases of respiratory failure (37).

\section{Post-treatment course - scoring systems}

In order to determine the optimal management course for a woman with obstetric complications, and to assist in deciding the group of patients that will require admission to CCUs of any kind, developing a prognostic and predictive model is crucial. Based on their utility in non-obstetric patients, established scoring systems, such as Acute Physiological and Chronic Health Evaluation (APACHE II), Sequel Organ Functional Assessment (SOFA), (Simplified Acute Physiology Score II (SAPS) and Mortality Prediction Model (MPM) have also been proposed for the evaluation of critically ill obstetric populations (40). According to el-Solh and Grant (41), the reported mortality rates as predicted by the APACHE II, SAPS II and MPM II scores for obstetric patients admitted to the ICU was comparable to those of non-obstetric patients of the same age. In addition to this, Aarvold et al (42) found no advantages to using the specific Sepsis in Obstetric Score for predicting the course of septic obstetric patients compared with the performance of the score in non-obstetric populations. Thus, whilst the exact roles 
of scoring systems in the prediction of mortality is still under investigation, and despite their broad use as a complementary tool, none of the scoring systems to date have proved useful. This is due to the fact that the physiological changes that occur during pregnancy, including increased heart rate, changes in white blood cell counts and decreased creatinine levels, can lead to higher calculated rates in the APACHE scoring system, and consequently an overestimated likelihood of mortality, and better outcomes than estimated using scoring systems have been reported by other studies $(40,43)$. In addition to this, the significant improvements that patients show following delivery in the majority of the parameters assessed is another critical factor that can complicate the estimation of the risk of death and post-delivery course (40). Finally, to the best of our knowledge, there are no studies that have compared the predictive value of the aforementioned scoring systems for patients in a CCU.

The Obstetric Early Warning Score(OEWS) has been reported to be the most accurate score for estimation of the outcomes of an obstetric patient in a CCU (44). The variables that are considered in the calculation of the new clinical OEWS consist of classical early warning signs, such as systolic and diastolic blood pressure, respiratory rate, heart rate, the $\mathrm{FiO} 2$ that is required to maintain $\mathrm{SpO}_{2}>96$ and body temperature in ${ }^{\circ} \mathrm{C}$, as well as the consciousness level with the mode of delivery (either caesarean or vaginal). According to the interpretation of OEWS, patients with a score of 0 will require routine care and normal observation, scores 1 and 2 correspond to yellow and red triggers, respectively, each highlighting a gradual increase in the severity of the disease $(44,45)$. The available evidence shows that this score is able to predict the survival of critically ill obstetric patients, which is also confirmed by the American College of obstetrics and gynecology and the Royal College of Obstetrics and Gynecology, which both suggest that OEWS is the best scoring system available for this particular patient population $(4,13)$. Despite the benefits of the OEWS system for the obstetric population, its impact on reducing mortality is still inconclusive and further studies are required (46). Despite the significant contribution of the aforementioned scores in the prediction of the course of patients admitted to CCUs, their role still remains complementary in the management of those patients, and the final decisions should combine the patients' and relatives' wishes, as well as the physicians judgement of the particular case (40).

Based on the currently available literature, the proportion of obstetric critically ill patients that are admitted to any kind of $\mathrm{CCU}$ is limited despite the fact that a significant proportion of pregnant women suffer from severe complications. That CCUs represent places with high quality care should not be neglected. Furthermore, the majority of patients admitted to CCUs for obstetrical complications require non-invasive monitoring and recovery support. The establishment of an intermediate care unit specialized for obstetric cases may thus be beneficial for these patients to alleviate or reduce stress (47). An experienced and specialized obstetrician should lead the unit and should be responsible for the final decisions with regard to the management of the patients, based on consultation of other specialists, including anesthesiologists and intensive care pathologists. Additionally, FMM specialists and neonatologists should also be enrolled in the multidisciplinary consultation team where possible. Establishment of a diagnostic and care algorithm based on the underlying pathology and the indication of admission, requires the contribution of such a multidisciplinary team, and is considered of critical importance for improving early designation of at risk patients to intensive care, and may also assist in deciding the type of CCU that each critically ill patient is admitted to (25). The aforementioned modalities may not only improve the level of care of all patients, but may also mitigate healthcare costs by reducing unnecessary admissions (47).

\section{Conclusions}

The management of critically ill obstetric patients remains a challenge due to the physiological changes that occur during pregnancy, the development of pregnancy-related diseases, and the need to carefully consider the well being of the fetus before any interventions to the mother can be performed. Indications for admission to CCUs include both obstetric and non-obstetric conditions that may require continuous monitoring and further interventions. Hypertensive disorders of pregnancy and massive hemorrhage are amongst the leading causes of admission to CCUs in pregnant and postpartum women. For the management of patients admitted to CCUs, the establishment of a diagnostic and care algorithm, based on the contributions of a multidisciplinary team including obstetricians/gynecologists, intensivists, anesthesiologists, FMM specialists and neonatologists is of critical importance to assist in determination of whether intensive care is quired and the appropriate type of CCU that each patient is admitted to, to ensure the safety of both the mother and the fetus.

\section{Acknowledgements}

Not applicable.

\section{Funding}

No funding was received.

\section{Availability of data and materials}

Not applicable.

\section{Authors' contributions}

NT conceived the topic of review. KK and AP searched the literature, and analyzed and interpreted the obtained articles. KK, AP and ES wrote the manuscript. NT and DV critically revised the manuscript. All authors have read and approved the final version of the manuscript. Data authentication is not applicable.

\section{Ethics approval and consent to participate}

Not applicable.

\section{Patient consent for publication}

Not applicable.

\section{Competing interests}

The authors declare that they have no competing interests to disclose. 


\section{References}

1. Minville V, Vidal F, Loutrel O, Castel A, Jacques L, Vayssière C, Parant $\mathrm{O}$, Guerby $\mathrm{P}$ and Asehnoune K: Identifying predictive factors for admitting patients with severe pre-eclampsia to intensive care unit. J Matern Fetal Neonatal Me: Sep 8, 2020 (Epub ahead of print). doi: 10.1080/14767058.2020.1814248.

2. Kazma JM, van den Anker J, Allegaert K, Dallmann A and Ahmadzia HK: Anatomical and physiological alterations of pregnancy. J Pharmacokinet Pharmacodyn 47: 271-285, 2020.

3. Baird SM and Martin S: Framework for critical care in obstetrics. J Perinat Neonatal Nurs 32: 232-240, 2018.

4. ACOG Practice Bulletin No: ACOG Practice Bulletin No. 211: Critical Care in Pregnancy. Obstet Gynecol 133: e303-e319, 2019.

5. Mgawadere F, Kana T and van den Broek N: Measuring maternal mortality: A systematic review of methods used to obtain estimates of the maternal mortality ratio (MMR) in low- and middle-income countries. Br Med Bull 121: 121-134, 2017.

6. Say L, Chou D, Gemmill A, Tunçalp Ö, Moller AB, Daniels J, Gülmezoglu AM, Temmerman M and Alkema L: Global causes of maternal death: A WHO systematic analysis. Lancet Glob Health 2: e323-e333, 2014.

7. Troiano NH and Witcher PM: Maternal mortality and morbidity in the United States: Classification, causes, preventability, and critical care obstetric implications. J Perinat Neonatal Nurs 32: 222-231, 2018.

8. Zieleskiewicz L, Chantry A, Duclos G, Bourgoin A, Mignon A, Deneux-Tharaux $\mathrm{C}$ and Leone M: Intensive care and pregnancy: Epidemiology and general principles of management of obstetrics ICU patients during pregnancy. Anaesth Crit Care Pain Med 35 (Suppl 1): S51-S57, 2016.

9. Hogan MC, Foreman KJ, Naghavi M, Ahn SY, Wang M Makela SM, Lopez AD, Lozano R and Murray CJ: Maternal mortality for 181 countries, 1980-2008: A systematic analysis of progress towards Millennium Development Goal 5. Lancet 375 $1609-1623,2010$

10. Souza JP, Cecatti JG, Haddad SM, Parpinelli MA, Costa ML, Katz L and Say L; Brazilian Network for Surveillance of Severe Maternal Morbidity Group; Brazilian Network for Surveillance of Severe Maternal Morbidity: The WHO maternal near-miss approach and the maternal severity index model (MSI): Tools for assessing the management of severe maternal morbidity. PLoS One 7: e44129, 2012.

11. Gaffney A: Critical care in pregnancy - is it different? Semin Perinatol 38: 329-340, 2014.

12. Pollock W, Rose L and Dennis CL: Pregnant and postpartum admissions to the intensive care unit: A systematic review. Intensive Care Med 36: 1465-1474, 2010.

13. Cantwell R, Clutton-Brock T, Cooper G, Dawson A, Drife J, Garrod D, Harper A, Hulbert D, Lucas S, McClure J, et al: Saving Mothers' Lives: Reviewing maternal deaths to make motherhood safer: 2006-2008. The Eighth Report of the Confidential Enquiries into Maternal Deaths in the United Kingdom. BJOG 118 (Suppl 1): $1-203,2011$

14. Edwards Z, Lucas DN and Gauntlett R: Is training in obstetric critical care adequate? An international comparison. Int J Obstet Anesth 37: 96-105, 2019.

15. Koukoubanis K, Bibasi V and Thomakos N: The foundamental role of postoperative critical care in gynecologic oncology surgery: A brief report. Ann Colorectal Res 8: 97-100, 2020.

16. Lee SW, Khaw KS, Ngan Kee WD, Leung TY and Critchley LA Haemodynamic effects from aortocaval compression at different angles of lateral tilt in non-labouring term pregnant women. Br J Anaesth 109: 950-956, 2012.

17. Ueland K, Novy MJ, Peterson EN and Metcalfe J: Maternal cardiovascular dynamics. IV. The influence of gestational age on the maternal cardiovascular response to posture and exercise. Am J Obstet Gynecol 104: 856-864, 1969.

18. Yeomans ER and Gilstrap LC III: Physiologic changes in pregnancy and their impact on critical care. Crit Care Med 33 (Suppl 10): S256-S258, 2005.

19. Contreras G, Gutiérrez M,Beroíza T, Fantín A, Oddó H, Villarroel L, Cruz E and Lisboa C: Ventilatory drive and respiratory muscle function in pregnancy. Am Rev Respir Dis 144: 837-841, 1991.

20. Pandya ST and Mangalampally K: Critical care in obstetrics. Indian J Anaesth 62: 724-733, 2018

21. Chesnutt AN: Physiology of normal pregnancy. Crit Care Clin 20: 609-615, 2004

22. Mushambi MC, Kinsella SM,Popat M,Swales H, Ramaswamy KK, Winton AL and Quinn AC; Obstetric Anaesthetists' Association; Difficult Airway Society: Obstetric Anaesthetists' Association and Difficult Airway Society guidelines for the management of difficult and failed tracheal intubation in obstetrics. Anaesthesia 70 1286-1306, 2015
23. Odutayo A and Hladunewich M: Obstetric nephrology: Renal hemodynamic and metabolic physiology in normal pregnancy. Clin J Am Soc Nephrol 7: 2073-2080, 2012.

24. Wiles K, Bramham K, Seed PT, Nelson-Piercy C, Lightstone L and Chappell LC: Serum Creatinine in Pregnancy: A Systematic Review. Kidney Int Rep 4: 408-419, 2018.

25. Einav $\mathrm{S}$ and Leone M: Epidemiology of obstetric critical illness. Int J Obstet Anesth 40: 128-139, 2019.

26. Jayaratnam S, Jacob-Rodgers S and de Costa C: Characteristics and preventability of obstetric intensive care unit admissions in Far North Queensland. Aust NZ J Obstet Gynaecol 60: 871-876, 2020.

27. Chawla S, Jose T and Paul M: Critical Care in Obstetrics: Where are We. J Obstet Gynaecol India 68: 155-163, 2018.

28. Dusse LM, Alpoim PN, Silva JT, Rios DR, Brandão AH and Cabral AC: Revisiting HELLP syndrome. Clin Chim Acta 451: 117-120, 2015.

29. Brown CE, Cunningham FG and Pritchard JA: Convulsions in hypertensive, proteinuric primiparas more than 24 hours after delivery. Eclampsia or some other cause? J Reprod Med 32: 499-503, 1987.

30. Guntupalli KK, Hall N, Karnad DR, Bandi V and Belfort M: Critical illness in pregnancy: part I: an approach to a pregnant patient in the ICU and common obstetric disorders. Chest 148: 1093-1104, 2015.

31. Siddiqui MM, Banayan JM and Hofer JE: Pre-eclampsia through the eyes of the obstetrician and anesthesiologist. Int J Obstet Anesth 40: 140-148, 2019.

32. Russell R: Preeclampsia and the anaesthesiologist: Current management. Curr Opin Anaesthesiol 33: 305-310, 2020.

33. Agarwal S and Laycock HC: The debate ROTEMs on - the utility of point-of-care testing and fibrinogen concentrate in postpartum haemorrhage. Anaesthesia 75: 1247-1251, 2020.

34. Gilmandyar D and Thornburg LL: Surgical management of postpartum hemorrhage. Semin Perinatol 43: 27-34, 2019.

35. Sorensen BL, Rasch V, Massawe S, Nyakina J, Elsass P and Nielsen BB: Advanced life support in obstetrics (ALSO) and post-partum hemorrhage: A prospective intervention study in Tanzania. Acta Obstet Gynecol Scand 90: 609-614, 2011.

36. Spreu A, Abgottspon F, Baumann MU, Kettenbach J and Surbek D: Efficacy of pelvic artery embolisation for severe postpartum hemorrhage. Arch Gynecol Obstet 296: 1117-1124, 2017.

37. Clark SL: Amniotic fluid embolism. Obstet Gynecol 123: 337-348, 2014.

38. Nawaz $\mathrm{N}$ and Buksh AR: Amniotic fluid embolism. J Coll Physicians Surg Pak 28: S107-S109, 2018.

39. Sultan P, Seligman K and Carvalho B: Amniotic fluid embolism: Update and review. Curr Opin Anaesthesiol 29: 288-296, 2016.

40. Gopalan PD and Muckart DJ: The critically ill obstetric patient: What's the score? Int J Obstet Anesth 13: 144-145, 2004.

41. el-Solh AA and Grant BJ: A comparison of severity of illness scoring systems for critically ill obstetric patients. Chest 110 : 1299-1304, 1996

42. Aarvold AB, Ryan HM, Magee LA, von Dadelszen P, Fjell C and Walley KR: Multiple organ dysfunction score is superior to the obstetric-specific sepsis in obstetrics score in predicting mortality in septic obstetric patients. Crit Care Med 45: e49-e57, 2017.

43. Vasquez DN, Estenssoro E, Canales HS, Reina R, Saenz MG, Das Neves AV, Toro MA and Loudet CI: Clinical characteristics and outcomes of obstetric patients requiring ICU admission. Chest 131: 718-724, 2007.

44. Carle C, Alexander P, Columb M and Johal J: Design and internal validation of an obstetric early warning score: Secondary analysis of the Intensive Care National Audit and Research Centre Case Mix Programme database. Anaesthesia 68: 354-367, 2013.

45. Umar A, Manu A, Mathai M and Ameh C: Development and validation of an obstetric early warning system model for use in low resource settings. BMC Pregnancy Childbirth 20: 531, 2020.

46. Shields LE, Wiesner S, Klein C, Pelletreau B and Hedriana HL: Use of maternal early warning trigger tool reduces maternal morbidity. Am J Obstet Gynecol 214: 527.e1-527.e6, 2016

47. Farr A, Lenz-Gebhart A, Einig S, Ortner C, Holzer I, Elhenicky M, Husslein PW and Lehner R: Outcomes and trends of peripartum maternal admission to the intensive care unit. Wien Klin Wochenschr 129: 605-611, 2017. 